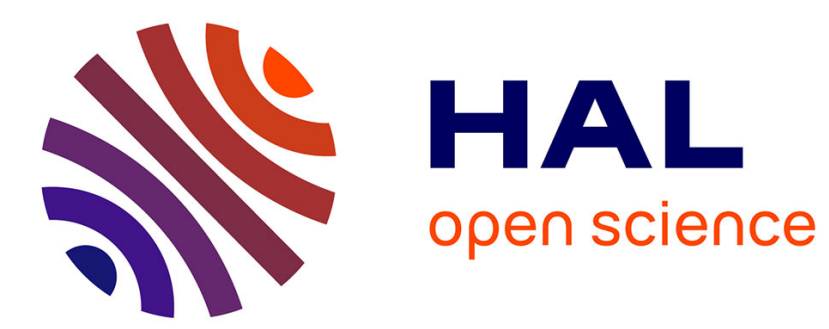

\title{
Loi d'échelle dans YBa2Cu3O7-x céramique
}

\author{
J. Soret, L. Ammor
}

\section{To cite this version:}

J. Soret, L. Ammor. Loi d'échelle dans YBa2Cu3O7-x céramique. Journal de Physique III, 1992, 2

(2), pp.203-212. 10.1051/jp3:1992118 . jpa-00248735

\section{HAL Id: jpa-00248735 \\ https://hal.science/jpa-00248735}

Submitted on 1 Jan 1992

HAL is a multi-disciplinary open access archive for the deposit and dissemination of scientific research documents, whether they are published or not. The documents may come from teaching and research institutions in France or abroad, or from public or private research centers.
L'archive ouverte pluridisciplinaire HAL, est destinée au dépôt et à la diffusion de documents scientifiques de niveau recherche, publiés ou non, émanant des établissements d'enseignement et de recherche français ou étrangers, des laboratoires publics ou privés. 
Classification

Physics Abstracts

74.70B

\title{
Loi d'échelle dans $\mathrm{YBa}_{2} \mathrm{Cu}_{3} \mathrm{O}_{7-x}$ céramique
}

\author{
J. C. Soret et L. Ammor
}

Université de Tours, Laboratoire de Physique Electronique et Thermodynamique des Oxydes, U.F.R. Sciences, 37200 Tours, France.

(Reçu le 7 novembre 1990, révisé le 16 juillet 1991, accepté le 10 septembre 1991)

\begin{abstract}
Résumé. - Nos mesures $V-I$ sur $\mathrm{YBa}_{2} \mathrm{Cu}_{3} \mathrm{O}_{7-x}$ céramique, dans la limite $I=0$, confirment une transition de phase du second ordre à une température critique $T_{c}(0)<T_{\mathrm{cs}}$ où $T_{\mathrm{cs}}$ est la température de condensation des grains. En présence d'un courant non nul la température de transition est déplacée à $T_{c}(I)<T_{c}(0)$. Nous montrons l'existence d'une température de crossover $T^{*}(I)$ dépendante du courant $I$. Pour les températures $T \gg T^{*}(I)>T_{\mathrm{c}}(0)$, $\sigma \sim \bar{t}^{-\gamma}$ avec $\gamma \simeq 0,9$ et $\bar{t}=\left(T-T_{\mathrm{c}}(I)\right) / T_{\mathrm{c}}(0)$, ce comportement est identique à celui observé en l'absence de courant $(I=0)$ lorsque $T \rightarrow T_{\mathrm{c}}(0)^{+}$. Pour les températures $T_{\mathrm{c}}(I) \leqslant T \ll T^{*}(I)<T_{\mathrm{c}}(0), \sigma \sim A(I) \bar{t}^{-\tilde{\gamma}}$ avec $\tilde{\gamma}=1,3$ et $A(I)=I^{(\bar{\gamma}-\gamma) \Phi}$
\end{abstract}

Abstract. - Our measurements $V-I$ in $\mathrm{YBa}_{2} \mathrm{Cu}_{3} \mathrm{O}_{7-x}$ ceramic, in the limit $I=0$, confirm a second-order phase transition at a critical temperature $T_{\mathrm{c}}(0)<T_{\mathrm{cs}}$ where $T_{\mathrm{cs}}$ is the critical temperature of the superconducting intragranular phase transition. As $I \neq 0$, we show the existence of a crossover temperature $T^{*}(I)$ depending on the value of the current $I$. For the temperatures $T \gg T^{*}(I)>T_{\mathrm{c}}(0), \quad \sigma \sim \bar{t}^{-\gamma}$ with $\gamma \simeq 0.9$ and $\bar{t}=\left(T-T_{\mathrm{c}}(I)\right) / T_{\mathrm{c}}(0)$ where $T_{\mathrm{c}}(I)$ corresponds to the critical temperature shift due to $I$. This conductivity critical behaviour is identical to the one observed as $T \rightarrow T_{\mathrm{c}}(0)^{+}$and $l=0$. For the temperature range $T_{\mathrm{c}}(I)<T \ll T^{*}(I)<T_{\mathrm{c}}(0) \sigma \sim A(I) \bar{t}-\tilde{\gamma}$ with $\tilde{\gamma}=1.3$ and $A(I)=I^{(\tilde{\gamma}-\gamma) \phi}$

\section{Introduction.}

L'observation d'une double anomalie dans la capacité calorifique autour de la transition de phase supra-conductrice dans $\mathrm{YBa}_{2} \mathrm{Cu}_{3} \mathrm{O}_{7-x}$ céramique [1-3] et monocristal $[4,5]$ a conduit certains auteurs à proposer l'existence d'une transition de phase à une température critique voisine de la température de transition $T_{\mathrm{cs}}$ de l'état normal à l'état supraconducteur (le lecteur pourra se reporter à la référence [6] pour une revue sur cette question). Plusieurs interprétations ont été proposées pour expliquer cette autre anomalie de la capacité calorifique : nouvelle phase supraconductrice [3], transition de phase du type structurale [5], augmentation de la supraconductivité aux joints de macle [7,8], coexistence en l'absence de champ magnétique appliqué de zones normales et supraconductrices [2] alors que les auteurs de la référence [1] ont cru observer l'anomalie de capacité calorifique prédite par Deutscher et al. [9] au seuil de percolation d'un supraconducteur granulaire. 
Très tôt après la découverte des oxydes supraconducteurs à températures critiques élevées, leur nature granulaire a été reconnue dans leur description phénoménologique [10]. Notamment, il a été montré [11] que ce concept est essentiel pour décrire la largeur finie de la transition résistive de ces matériaux comme résultant de la transition supraconductrice intragranulaire à $T_{\mathrm{cs}}$ suivie d'une transition du second ordre à la cohérence de phase entre grains à $T_{\mathrm{c}}(0)<T_{\mathrm{cs}}$ [12]. Il est attendu [13] et il a été montré [14] que les propriétés de transport obéissent à une loi d'échelle à la transition vers la cohérence de phase.

A partir de mesures $V-I$, en l'absence de champ magnétique extérieur, obtenues à des températures $T<T_{\mathrm{cs}}$ nous avons déterminé une transition de phase du second ordre à une température critique $T_{\mathrm{c}}(I)<T_{\mathrm{cs}}$. La partie 2 décrit la méthode expérimentale. La partie 3 présente les résultats expérimentaux montrant l'existence d'une loi d'échelle entre la tension et le courant, laquelle définit deux exposants critiques. Les formes asymptotiques des deux fonctions de similitude sont similaires à celles prédites par ailleurs [13]. En particulier dans cet article, nous insistons sur le déplacement de la température critique $T_{c}(l)$ avec le courant $I$ et nous montrons que la même loi d'échelle décrit le changement de comportement critique de la conductivité électrique autour d'une température de crossover $T^{*}(I)$ dépendante du courant $I$.

\section{Méthode expérimentale.}

Notre échantillon de géométrie cylindrique (hauteur $\sim 30 \mathrm{~mm}$, diamètre $\sim 5 \mathrm{~mm}$ a été préparé à partir des composés de départ $\mathrm{Y}_{2} \mathrm{O}_{3}, \mathrm{Ba}\left(\mathrm{CO}_{3}\right)_{2}$ et $\mathrm{CuO}$ dissous en proportions stœchiométriques $1 / 2,2,3$ dans l'acide nitrique puis coprécipités par l'acide oxalique. Après calcination la poudre est compactée et frittée sous air à $900^{\circ} \mathrm{C}$ puis refroidie lentement jusqu'à $250^{\circ} \mathrm{C}$. Une analyse par diffraction de rayon $\mathrm{X}$ a confirmé la présence d'une seule phase correspondant à la phase orthorhombique de la structure pérovskite. Le comportement résistif $\rho(T)$ est du type métallique avec $\rho(300) \sim 6 \times 10^{-6} \Omega \mathrm{m}$ et $\rho(300) / \rho\left(T_{\mathrm{cs}}\right) \sim 1,4$. Les mesures électriques ont été effectuées dans un cryostat à azote blindé par une chambre en mumétal de tout champ magnétique extérieur. La température $T$ de l'échantillon était régulée avec une stabilité de $0,05 \mathrm{~K}$ entre $77 \mathrm{~K}$ et les températures au-dessus de $T_{\text {cs. Nous avons }}$ déterminé les caractéristiques $V-I$ en régime continu par la méthode des 4 fils. La résolution sur la mesure de la tension était de $5 \mathrm{nV}$. Nous avons réalisé de larges contacts d'amenée de courant, recouvrant chacune des extrémités de l'échantillon sur une longueur de $5 \mathrm{~mm}$, par dépôt de laque d'argent suivi d'un traitement thermique ce qui nous a permis d'obtenir une résistance de contact de l'ordre de $10^{-2} \Omega \mathrm{cm}^{2}$.

\section{Résultats et discussion.}

La figure 1 montre les caractéristiques $V-I$ de l'échantillon pour différentes températures. Suivant la valeur du courant, il peut être distingué trois domaines. Le premier correspond au régime de conduction parfaite (absence de dissipation pour $I<I_{\mathrm{c}}(T)$ où $I_{\mathrm{c}}(T)$ définit le courant critique). La figure 2 montre de façon qualitative que le régime de conduction parfaite disparaît pour les températures supérieures à $87,15 \mathrm{~K}<T_{\mathrm{c}}(0)<88,05$ où $T_{\mathrm{c}}(0)$ est solution de l'équation $I_{\mathrm{c}}\left[T_{\mathrm{c}}(0)\right]=0$. Le second domaine en courant est caractérisé par le comportement non ohmique de l'échantillon alors que dans le troisième domaine la résistance de l'échantillon est celle mesurée dans l'état normal. La figure 1 montre que le basculement du second domaine au troisième domaine lors de la croissance du courant est de moins en moins brusque lorsque la température croît. Nous remarquons que le second domaine en courant disparaît à une température $91 \mathrm{~K}<T_{\mathrm{cs}}<93 \mathrm{~K}$. La température $T_{\mathrm{cs}}$ correspond à la 


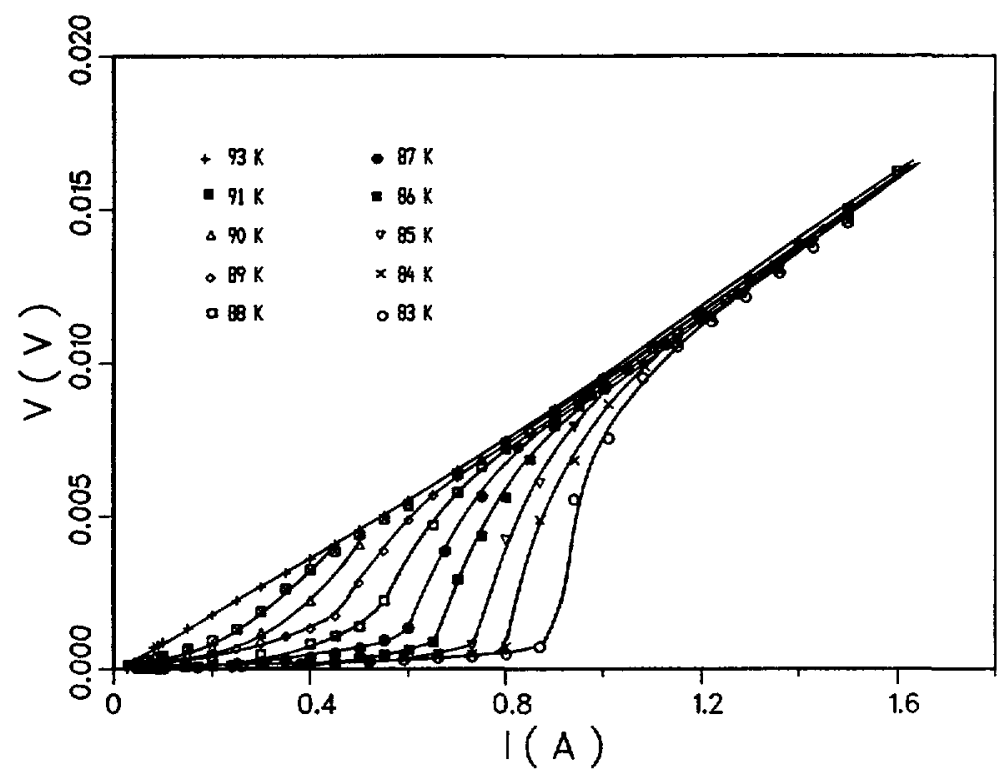

Fig. 1. - Caractéristiques tension-courant de l'échantillon.

[Induced voltage $V$ versus transport current $I$ with zero applied magnetic field.]

température que l'on a l'habitude d'appeler la $T_{\mathrm{c}}$ « onset » de la courbe de résistance en fonction de la température.

Dans cet article nous nous intéresserons uniquement aux premier et second domaines en courant, c'est-à-dire aux caractéristiques $V-I$ montrées sur la figure 2.

Nous avons déterminé le courant critique à partir de la forme

$$
V \sim\left(I-I_{\mathrm{c}}(T)\right)^{\xi}
$$

Ce comportement hypothétique de la tension près de $I_{\mathrm{c}}(T)$ est pratique car il permet de déterminer le courant critique sans avoir recours à un critère arbitraire du type tension de seuil [15]. Nous reviendrons par la suite sur la validité de (1). Pratiquement, pour déterminer $I_{\mathrm{c}}(T)$ à partir de (1), nous avons utilisé une méthode de moindres carrés pour le lissage de $V^{1 / \xi}$ en fonction de $I$ pour les caractéristiques $V-I$ à $T<87,15 \mathrm{~K}$ de la figure 2 . L'exposant $\xi$ est ajusté pour obtenir la meilleure droite. Alors la prolongation $V^{1 / \xi}=0$ donne $I_{\mathrm{c}}(T)$. Cependant, pour que cette méthode soit valable, nous devons vérifier que la valeur optimale de l'exposant $\xi$ soit peu sensible aux points expérimentaux de plus hautes valeurs en courant. Autrement dit l'exposant $\xi$ ne doit pas être une fonction du courant $I$.

Sur la figure 3, nous avons tracé les valeurs ajustées de l'exposant $\xi$ en fonction de la température. Il peut être remarqué que $\xi$ reste sensiblement constant, $\xi=2,3$, jusqu'à une température de l'ordre de $85 \mathrm{~K}$. Au-dessus de cette température l'exposant $\xi$ dépend du courant $I$ et notre méthode de calcul n'est plus exploitable. Cependant nous pensons que la dépendance de $\xi$ avec $l$ indique que la relation (1) n'est plus valable à ces températures pour les niveaux de courant utilisés (voir plus bas). Par conséquent, à partir de (1), nous n'avons pu déterminer les valeurs du courant critique $I_{\mathrm{c}}(T)$ que seulement pour les températures inférieures à $85 \mathrm{~K}$. Nous avons recherché une loi de puissance du type $I_{c}(T) \sim\left(T_{c}(0)-T\right)^{\Phi}$ 


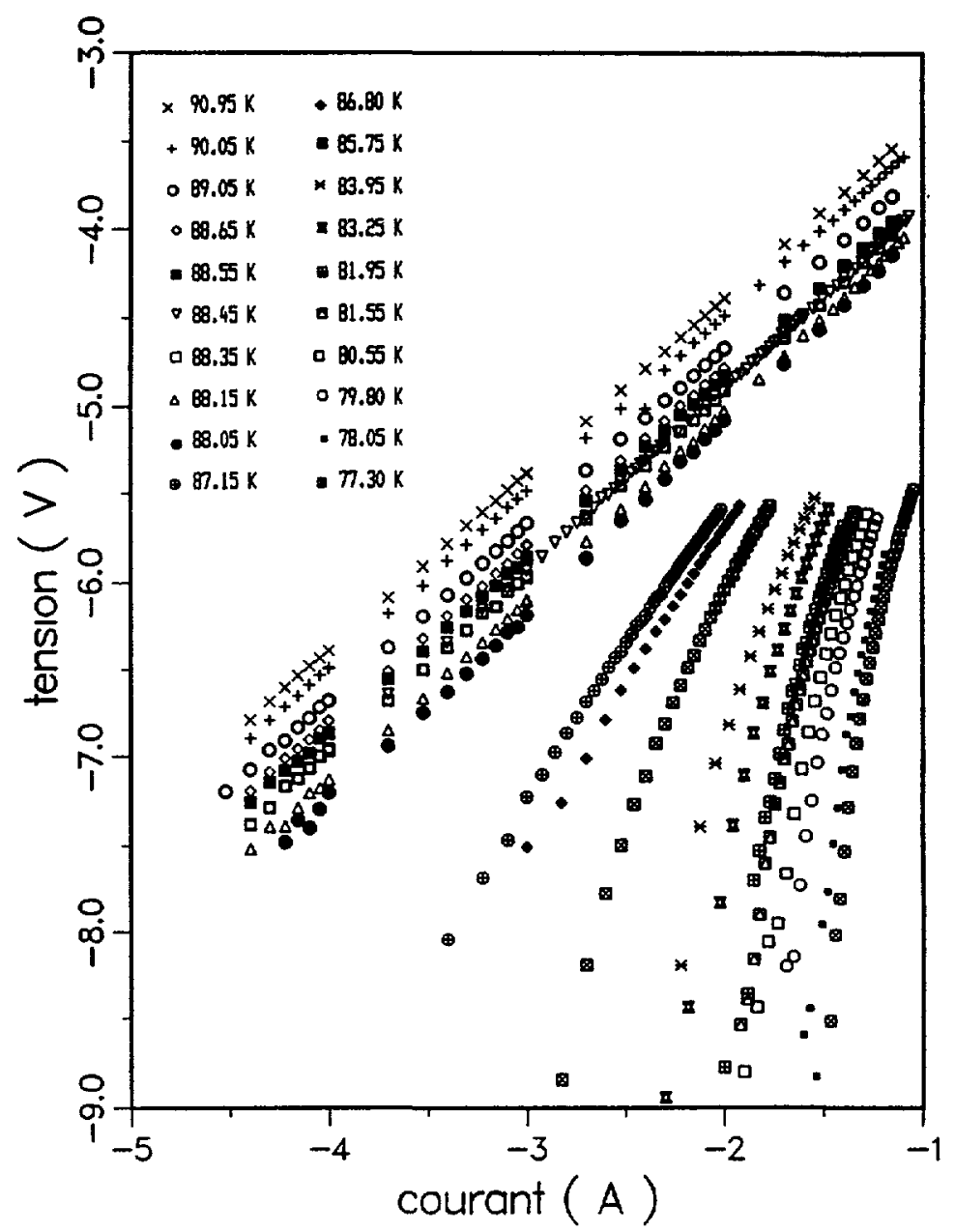

Fig. 2. - Caractéristiques tension-courant pour les valeurs du courant $I$ inférieures à $100 \mathrm{~mA}$.

[Logarithmic plot of induced voltage $V$ versus transport current $I<100 \mathrm{~mA}$ ].

La figure 4 montre en représentation logarithmique $I_{\mathrm{c}}$ en fonction $\left(T_{\mathrm{c}}(0)-T\right) / T_{\mathrm{c}}(0)$ où $T_{\mathrm{c}}(0)=87,7 \mathrm{~K}$ nous a permis d'obtenir la meilleure droite. Suivant la figure 4 nous déduisons que les valeurs du courant critique en fonction de la température sont bien représentées par la loi de puissance $I_{\mathrm{c}}(T) \sim\left(T_{\mathrm{c}}(0)-T\right)^{1,8}$ où la valeur de l'exposant est donnée par la pente de la droite. La prolongation de cette loi conduit à l'annulation du courant critique $I_{c}$ à une température $T_{\mathrm{c}}(0)$ inférieure à la température de transition $T_{\mathrm{cs}}$ vers l'état normal comme nous l'annoncions de façon qualitative précédemment. Une telle conduite a déjà été mise en évidence dans : $\mathrm{YBa}_{2} \mathrm{Cu}_{3} \mathrm{O}_{7-x}[2,11]$ et elle est bien connue dans les supraconducteurs granulaires où $T_{\mathrm{c}}(0)$ apparaît comme une transition de phase résistive du second ordre [12] autour de laquelle il est prévu que les propriétés de transport obéissent à une loi d'échelle [13].

Sur la figure 5 , nous avons tracé $V / t^{\mu}$ en fonction de $I / t^{\Phi}$ où $t$ représente la température réduite $\left|T-T_{\mathrm{c}}(0)\right| / T_{\mathrm{c}}(0), \Phi$ est l'exposant du courant critique $\mu$ a été choisi égal à 2,7 . 


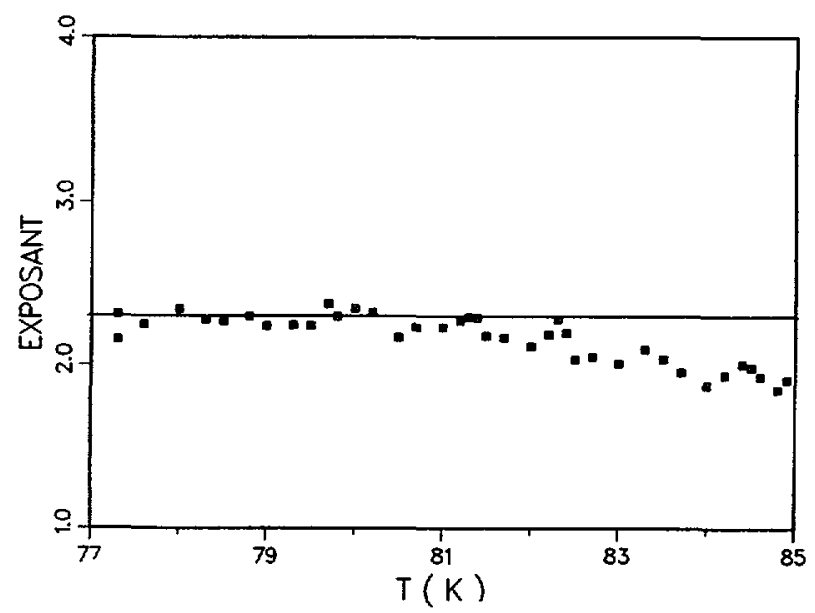

Fig. 3. - Valeurs de l'exposant $\xi$ calculées à partir de la relation (1) en fonction de la température $T$. Le trait horizontal indique la valeur $\xi=2,3$.

[Values of the exponent $\xi$ calculated from relation (1) as a function of temperature $T$. The horizontal line indicates the value $\xi=2.3$.

Cette valeur de l'exposant $\mu$ est plutôt serrée pour obtenir une relation indépendante de la température entre $V / t^{\mu}$ et $I / t^{\Phi}$

$$
V / t^{\mu}=f_{ \pm}\left(I / t^{\Phi}\right)
$$

Respectivement, les fonctions de similitude $f_{+}$et $f_{-}$sont associées au domaine des températures supérieures à $T_{\mathrm{c}}(0)$ et au domaine des températures inférieures à $T_{\mathrm{c}}(0)$. Les deux exposants $\mu$ et $\Phi$ possèdent un caractère universel dans le sens qu'ils restent constants au-dessus et au-dessous de la température de transition $T_{\mathrm{c}}(0)$. Précédemment, dans le cas de films supraconducteurs granulaires $(\mathrm{NbN}, \mathrm{Hg}-\mathrm{Xe})$, une telle loi expérimentale de similitude entre $V$ et $I$ a été observée et l'analogie avec l'équation d'état, valable dans le voisinage de la transition de phase, d'un système magnétique a été faite [16, 17].

A partir de la figure 5, il peut être déduit que le courant $I$ apparaît comme un paramètre pertinent $[18,19]$ pour la transition de phase à $T_{\mathrm{c}}(0)$. Deux domaines peuvent être remarqués au-dessus de la température de transition $T_{\mathrm{c}}(0)$ suivant la valeur de $z=I / t^{\Phi}$ Dans le premier correspondant à $z<10$, la fonction d'échelle $f_{+}(z)$ se comporte comme $f(z) \sim z$ et par conséquent à partir de (2), il est facile de déduire que la conductivité se conduit comme $\sigma \sim t^{-\gamma}$ avec l'exposant $\gamma=\mu-\Phi=0,9$. Cependant, dans le second domaine associé aux valeurs $z \gg 10$ les effets non linéaires dominent. A la limite $z \rightarrow \infty$, c'est-à-dire $T \rightarrow T_{\mathrm{c}}(0)^{+}$ avec $I \neq 0$, le comportement asymptotique de la fonction $f_{+}(z)$ est $z^{\mu / \Phi}[13,14]$ avec $\mu / \Phi=3 / 2$. Par conséquent, il peut être déduit à partir de (2) que $V \sim I^{3 / 2}$ à $T_{\mathrm{c}}(0)$. Ainsi, lorsqu'un courant $I$ est imposé à travers l'échantillon, il existe une température assez bien définie mais dépendante de la valeur du courant $I$ au-dessus de laquelle ce dernier apparaît comme une faible perturbation, c'est-à-dire le comportement de la conductibilité est identique au cas $I=0$ et $t \neq 0$, et au-dessous de laquelle le courant $I$ apparaît comme une forte perturbation conduisant à la disparition de la transition de phase résistive à $T_{\mathrm{c}}(0)$. 


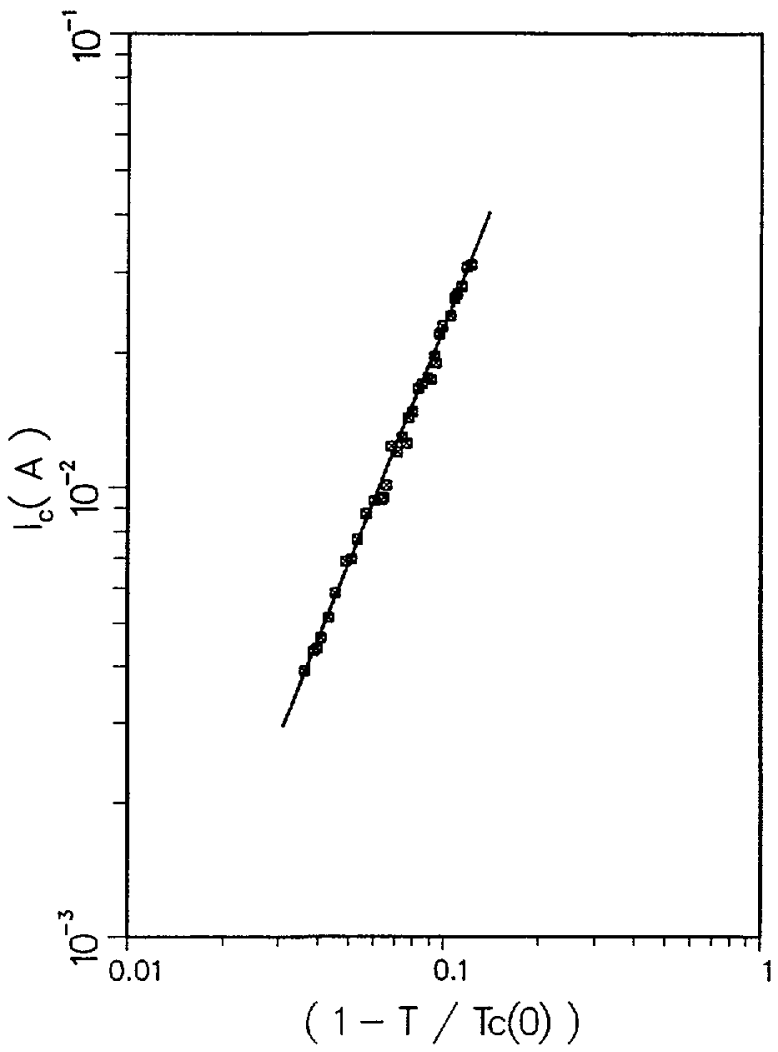

Fig. 4. - Tracé logarithmique de $I_{\mathrm{c}}$ calculé à partír de la relation (1) en fonction de la température réduite $\left(T_{\mathrm{c}}(0)-T\right) / T_{\mathrm{c}}(0)$ où $T_{\mathrm{c}}(0)=87,7 \mathrm{~K}$ est choisie pour obtenir la meilleure droite. La pente de la droite est égale à 1,8 .

[Logarithmic plot of critical current $I_{\mathrm{c}}$ calculated from relation (1) as a function of reduced temperature $\left(T_{\mathrm{c}}(0)-T\right) / T_{\mathrm{c}}(0)$ where $T_{\mathrm{c}}(0)=87.7 \mathrm{~K}$ produces the best straight line. The slope is equal to 1.8.]

Au-dessous de la température $T_{\mathrm{c}}(0)$, la fonction d'échelle $f_{-}(z)$ pour $z \rightarrow \infty$ a le même comportement asymptotique que $f_{+}(z)$ et est identiquement nulle au-dessous d'une valeur particulière $z_{\mathrm{c}}$. Nous avons voulu lisser la fonction de similitude $f_{-}(z)$ par une loi de puissance du type $f_{-}(z) \sim\left(z-z_{\mathrm{c}}\right)^{\xi^{\prime}}$ A cette fin, nous nous sommes servi de la méthode des moindres carrés utilisée pour la détermination de $I_{c}(T)$ à partir de la relation (1) et décrite précédemment. Sur la figure 5 , la ligne en pointillé représente le meilleur lissage obtenu et correspond à $f_{-}(z) \sim\left(z-z_{\mathrm{c}}\right)^{2,3}$ avec $z_{\mathrm{c}}=1,6$.

Il est intéressant de noter que la valeur calculée de l'exposant $\xi^{\prime}$ coïncide avec l'exposant $\xi$ de la relation (1) - voir la figure 3. Cependant, il est évident que le lissage de la fonction de similitude $f_{-}(z)$ par la loi $\left(z-z_{\mathrm{c}}\right)^{2,3}$ ne peut pas rendre compte du comportement asymptotique $f_{-}(z) \sim z^{3 / 2}$; par conséquent ce lissage ne pourra être correct qu'au voisinage de $z_{\mathrm{c}}$. Ainsi la relation (1) apparaît valable seulement au-dessous d'une valeur du courant $I^{*}$ laquelle dépend de la température comme $I^{*}(T) \sim t^{\Phi}$. Autrement dit, la résolution finie de nos mesures en tension ne nous permet pas d'imposer des courants $I<I^{*}(T$ ) (dans la limite de notre générateur de courant) et nous place en dehors du domaine en courant où la relation (1) est valable lorsque la température $T \rightarrow T_{\mathrm{c}}(0)^{-}(t \rightarrow 0)$. De plus la limite 


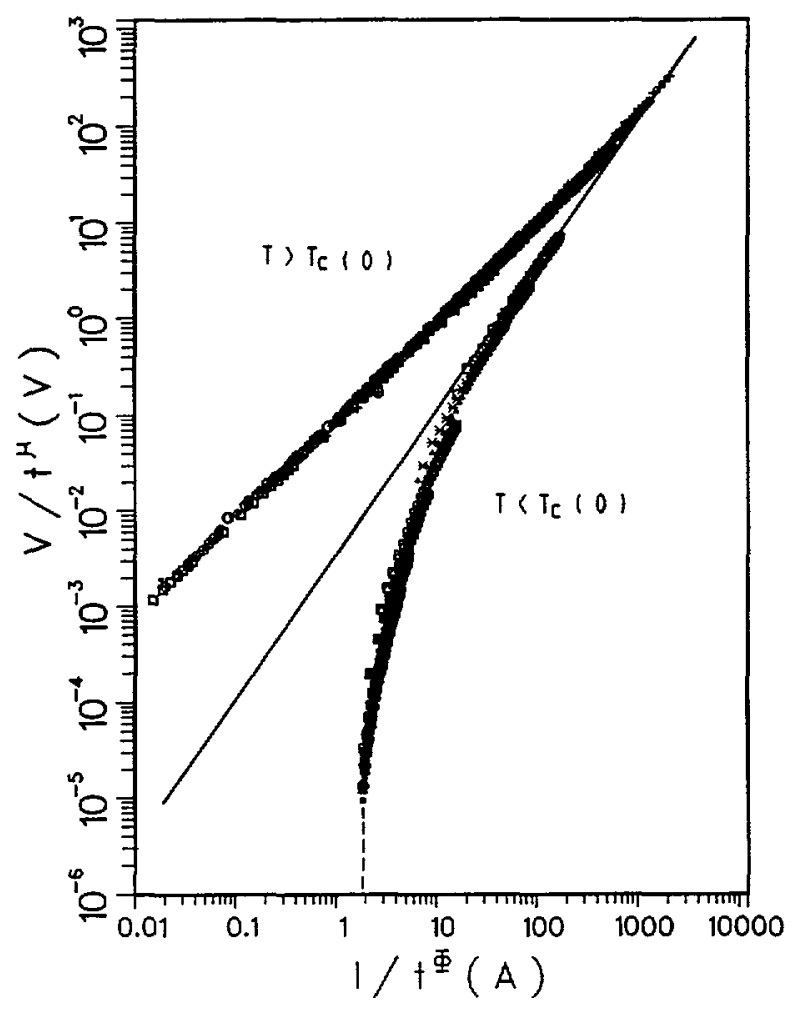

Fig. 5. - Tracé logarithmique de $V / t^{\mu}$ en fonction de $I / t^{\Phi}$. $\Phi$ est l'exposant du courant critique et $\mu=2,7$. Ces points sont à comparer avec ceux de la figure 2. A l'asymptote les deux branches se comportent comme $V / t^{\mu} \sim\left(I / t^{\Phi}\right)^{3 / 2}$ Pour $I / t^{\Phi}<10$, la branche supérieure est linéaire $V / t^{\mu} \sim I / t^{\Phi}$. La ligne en pointillé est le résultat du lissage de la branche inférieure par $\left(I / t^{\Phi}-z_{\mathrm{c}}\right)^{2,3}$

[Logarithmic plot of $V / t^{\mu}$ versus $z=1 / t^{\Phi}$. $\Phi$ is the critical current exponent and $\mu=2.7$. The symbols are those of figure 2 . The two branches $f_{ \pm}$are asymptotic to the solid straight line which corresponds to $V \sim I^{3 / 2}$ In the domain corresponding to $z<10 f_{+}(z) \sim z$. The dashed line exhibits the fit $f_{-}(z) \sim\left(z-z_{c}\right)^{2.3}$ with $\left.z_{c}=1.6.\right]$

$f_{-}(z) \rightarrow 0$ pour $z \rightarrow z_{\mathrm{c}}$ confirme a postériori la validité du prolongement de l'expression du courant critique, $I_{\mathrm{c}}(T)=z_{\mathrm{c}} t^{\Phi}$

A partir de l'expression de la fonction d'échelle $f_{-}(z) \sim\left(z-z_{c}\right)^{2,3}$ correcte près de $z_{\mathrm{c}}$ et de la relation (2) il est facile de vérifier que la conductivité est parfaite aux températures $T<T_{\mathrm{c}}(I)<T_{\mathrm{c}}(0)$ où $T_{\mathrm{c}}(I)$ est solution de l'équation $I / t_{\mathrm{c}}^{\Phi}=z_{\mathrm{c}}$ [16]. Ainsi le courant $I$ déplace la température de la transition de phase résistive de $T_{\mathrm{c}}(0)$ à $T_{\mathrm{c}}(I)$.

A ce stade, il est intéressant de voir comment le comportement critique autour de $T_{\mathrm{c}}(0)$ disparaît en présence d'un courant $I$ et s'il fait place à un comportement critique différent à $T_{\mathrm{c}}(I)$. L'équation $I / t^{\Phi}=z_{\mathrm{c}}$ définissant $T_{\mathrm{c}}(I)$ montre que la température de la transition en fonction du courant se déplace comme $T_{\mathrm{c}}(0)-T_{\mathrm{c}}(I) \sim I^{1 / \Phi}$ ce qui permet de supposer une même fonction d'échelle pour décrire le passage d'un comportement critique à un autre $[18,19]$. Dans ce cas il est intéressant d'utiliser la température réduite $\bar{t}=\left(T-T_{\mathrm{c}}(I)\right) / T_{\mathrm{c}}(0)$. 
Sur la figure 6, nous avons tracé $\sigma / \bar{t}^{-\gamma}$ en fonction de $\left(I / z_{\mathrm{c}}\right) / \bar{t}^{\Phi}$ où $\gamma=\mu-\Phi$. la valeur des exposants $\Phi$ et $\mu$ est inchangée par rapport à la figure 5. La conductivité $\sigma$ est calculée à partir de la pente des caractéristiques $V-I$ de la figure 2 .

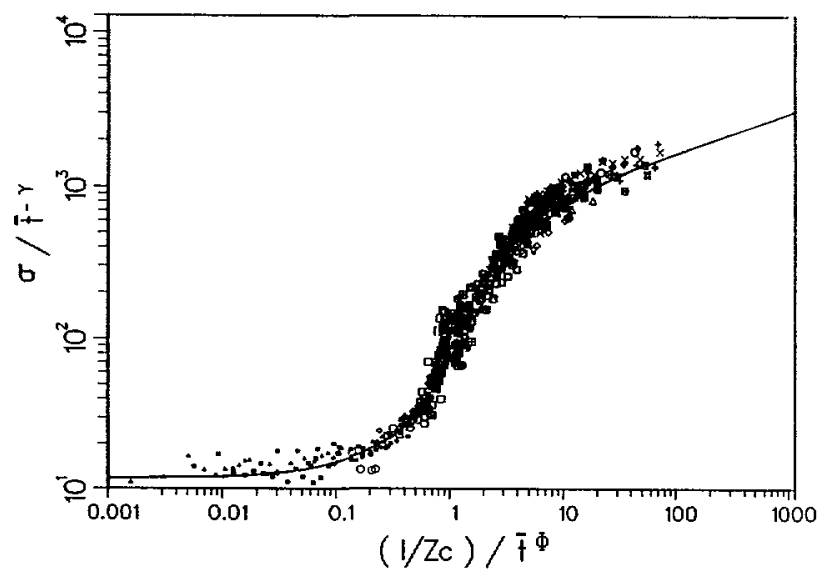

Fig. 6. - Tracé logarithmique de $\sigma / \bar{t}^{-\gamma}$ en fonction de $\left(I / z_{\mathrm{c}}\right) / \bar{t}^{\Phi}$ Les exposants $\gamma=\mu-\Phi$ et $\Phi$ sont ceux de la figure 5. Les différents symboles se rapportent à ceux de la figure 2 . Les deux lignes continues sont obtenues respectivement à partir du comportement linéaire de la branche supérieure et du lissage de la branche inférieure de la figure 5.

[Logarithmic plot of $\sigma / \bar{t}^{-\gamma}$ versus $\bar{z}=\left(I / z_{\mathrm{c}}\right) / \bar{t}^{\Phi}$ with $\gamma=\mu-\Phi$ and $\bar{t}=\left(T-T_{\mathrm{c}}(I)\right) / T_{\mathrm{c}}(0)$. The exponents $\mu$ and $\Phi$ are those of figure 5. The symbols are those of figure 2 . Respectively, the two solid lines are obtained from linear part of $f_{+}(z)$ and from $\left.f_{-}(z) \sim\left(z-z_{\mathrm{c}}\right)^{23}\right]$

Les valeurs $\bar{z}=\left(I / z_{\mathrm{c}}\right) \bar{t}^{\Phi}$ respectivement supérieures et inférieures à l'unité sont associées aux températures inférieures et supérieures à $T_{\mathrm{c}}(0)$; la valeur $\bar{z} \rightarrow \infty$ est atteinte lorsque $T \rightarrow T_{\mathrm{c}}(I)^{+}$alors que la valeur $\bar{z}=0$ est réalisée lorsque $I=0\left(T>T_{\mathrm{c}}(0)\right)$. La figure 6 montre une relation de similitude entre $\sigma / \bar{t}^{-\gamma}$ et $\bar{z}$ :

et

$$
\sigma / \bar{t}^{-\gamma}=\chi(\bar{z}) \text { où } \bar{z}=\left(I / z_{\mathrm{c}}\right) / \bar{t}^{\Phi}
$$

Les lignes continues respectivement au-dessous et au-dessus de la valeur $\bar{z}=1$ ont été calculées en accord avec le comportement linéaire de $f_{+}(z)$ et l'expression de $f_{-}(z) \sim\left(z-z_{\mathrm{c}}\right)^{2,3}$ valable près de $z_{\mathrm{c}}$. Nous pouvons vérifier avec l'œil que le raccordement du prolongement de ces deux lignes à travers la frontière $\bar{z}=1, T=T_{\mathrm{c}}(0)$, ne fait apparaître aucune singularité. La fonction $\chi(\bar{z})$ est constante pour les faibles valeurs de $\bar{z}$ alors qu'elle se comporte comme $\chi(\bar{z}) \sim \bar{z}^{a}$ avec $a=0,25$ pour les grandes valeurs de $\bar{z}$. Ainsi, à partir de la relation (3), nous déduisons les deux comportements suivants :

$$
\begin{array}{rrr}
\sigma \sim \bar{t}^{-\gamma} & \text { avec } & \gamma=0,9 \\
\sigma \sim A(I) \bar{t}^{-\tilde{\gamma}} & \text { avec } & \tilde{\gamma}=1,3
\end{array}
$$

de la conductivité valables respectivement dans les intervalles' $\bar{z} \ll 1$ et $\bar{z} \gg 1$. Dans la 
relation (5), le terme multiplicateur $A(I)$ est une fonction ayant l'expression $A(I)=I^{(\tilde{\gamma}-\gamma) / \Phi}$. Il est intéressant de noter que la relation (4) est valable pour $\bar{z}=0$ et qu'elle redonne alors le comportement critique de la conductivité au-dessus de la transition de phase résistive à $T_{\mathrm{c}}(0)$ en l'absence de courant $(I=0)$.

De façon claire, les relations (4) et (5) décrivent le passage d'un comportement critique à un autre pour la conductivité où le courant $I$ joue le rôle d'un champ extérieur, l'exposant $\Phi>0$ apparaissant comme un exposant de changement de comportement critique [18, 19]. Nous pouvons noter, d'après la figure 6 , que ce changement de comportement critique s'effectue dans le voisinage d'une température assez bien définie $T^{*}(l)$ dépendant de la valeur du courant $I$ traversant l'échantillon. Lorsque la température $T$ s'approche de $T_{\mathrm{c}}(I)$ trois domaines de température apparaissent. Dans le premier $T \gg T^{*}(I)>T_{\mathrm{c}}(0)$ tout se passe comme si le courant $I$ était nul; dans le second, à partir de la température de crossover $T^{*}(I)$, le courant $I$ fait disparaître le comportement critique de la conductivité caractérisé par l'exposant $\gamma$ pour faire apparaître dans le troisième domaine $T_{\mathrm{c}}(I) \leqslant T \ll T_{\mathrm{c}}(0)<T^{*}(I)$ un second comportement critique de la conductivité caractérisé par l'exposant $\tilde{\gamma}>\gamma$.

\section{Conclusion.}

Dans cet article, nous confirmons l'existence d'une transition de phase du second ordre à une température $T_{\mathrm{c}}(0)$ inférieure à la température de transition à l'état supraconducteur des grains dans $\mathrm{YBa}_{2} \mathrm{Cu}_{3} \mathrm{O}_{7-x}$ céramique.

Nous mettons en évidence une loi d'échelle entre la conductivité et le courant 1. Cette loi définit deux exposants critiques $\gamma$ et $\Phi>0$. Le premier caractérise la divergence de la conductivité lorsque $T \rightarrow T_{c}(0)^{+}$en l'absence de courant $(I=0)$ et le second lié au courant $I$ apparaît comme un exposant de changement de comportement critique.

En présence d'un courant $I \neq 0$, la température de transition est déplacée à $T_{\mathrm{c}}(I)$ et nous montrons que la même loi d'échelle décrit le basculement progressif d'un comportement critique à un autre au voisinage d'une température de crossover $T^{*}(I)$ dépendant du courant I. Dans la région $T \gg T^{*}(I)>T_{\mathrm{c}}(0)$ le comportement de la conductivité est donné par la relation [4] alors que la relation [5] est valable pour les températures $T_{\mathrm{c}}(I) \leq T \ll T_{\mathrm{c}}(0)<T^{*}(I)$. Si l'on suppose $L \gg \lambda(T) \sim t^{-\beta}$ ( $L$ : taille de l'échantillon) l'exposant critique $\varphi$ de la densité de courant critique $\left(j_{\mathrm{c}} \sim \frac{I_{\mathrm{c}}}{\lambda} \sim t^{\varphi}\right)$ est égal à $\varphi=\Phi+\beta$. En choisissant $\beta \simeq 0,7$ [14] $\varphi \simeq 2,5$. Cette valeur, proche de celle $(\varphi \simeq 2,66)$ prédite dans la zone critique d'un supraconducteur granulaire [14], est compatible avec une large région critique $(t \sim 0,1)$ attendue dans les oxydes supraconducteurs à hautes températures critiques [20].

Le paramètre d'ordre de cette transition de phase ne peut pas être lié directement au courant $I$ (comme l'aimantation d'un système magnétique est lié au champ magnétique) car il est connu que la présence d'un champ extérieur conjugué du paramètre d'ordre conduit à la disparition de la transition de phase. Ainsi le paramètre d'ordre de cette transition ne semble pas directement mesurable à partir de mesures électriques.

\section{Remerciements.}

Nous remercions les Laboratoires de Marcoussis-C.G.E. pour la fourniture de l'échantillon étudié. 


\section{Bibliographie}

[1] Li F. et al., Solid State Commun. 64 (1987) 209.

[2] Butera R. A., Phys. Rev. B 37 (1988) 5909.

[3] Ishikawa N. et al., Physica C 153-155 (1988) 1089.

[4] INDERheEs S. E. et al., Phys. Rev. Lett. 60 (1988) 1178.

[5] KE-QIN W. et al., Solid State Commun. 69 (1989) 1145.

[6] ChOY T. C. et al., Phase Transitions 20 (1990) 1.

[7] Sobyanin A. A. et al., Physica C 153-155 (1981) 1681.

[8] Abrikosov A. A. et al., Supercond. Sci. Technol. 1 (1989) 260.

[9] Deutscher G. et al., Phys. Rev. B 21 (1980) 5041.

[10] Múller K. A. et al., Phys. Rev. Lett. 58 (1987) 1143.

[11] LeBeAU C. et al., Proceedings of the International Conference Modern Aspects of Superconductivity (Paris, 1989) pp. 179.

[12] Pellan P. et al., Solid State Commun. 11 (1972) 427.

[13] LebeaU C. et al., Europhys. Lett. 1 (1986) 313.

[14] Peyral P. et al., J. Less Common Metals 151 (1989) 49.

[15] DE LA CRuz F. et al., Phys. Rev. B 36 (1987) 6850.

[16] Wolf S. A. et al., Phys. Rev. Lett. 42 (1979) 324.

[17] EPSTEIN K. et al., Physica B 107 (1981) 323.

[18] Boccara N., Symétries brisées (publié chez (Hermann, 1976) avec le concours du C.N.R.S.).

[19] TOUlouse G., PFEuTY P., Introduction au groupe de renormalisation et à ses applications (Presses Universitaires de Grenoble, 1975).

[20] Loвв C. J., Phys. Rev. B 36 (1987) 3930. 


\title{
Investigation de divers procédés de texturation de céramiques supraconductrices à haute $\boldsymbol{T}_{\mathrm{c}}$
}

Pierre Régnier ( $\left.{ }^{1}\right)$. Xavier Deschanels ( $\left.{ }^{1}\right)$, Laurent Chaffron $\left({ }^{1}\right)$, Lélia Schmirgeld ( ${ }^{1}$, Pol Godelaine ( $\left.{ }^{2}\right)$, Marcel Ausloos $\left({ }^{2}\right)$, Hyacinthe Vanderschueren $\left({ }^{2}\right)$, Robert Pollard $\left({ }^{3}\right)$, Graham McCartney $\left({ }^{3}\right)$, Clotilde Aguillon $\left({ }^{4}\right)$, Sadok Senoussi $\left({ }^{4}\right)$, Philippe Monod $\left({ }^{4}\right)$ et Nicole Bontemps $\left({ }^{4}\right)$

(1) DTA/CEREM/DTM/SRMP-CE Saclay, 91191 Gif-sur-Yvette Cedex, France

(2) Montefiore Univ. de Liège, Sart Tilman B-28, B 4000 Liège, Belgique

(3) M.S.E. The University of Liverpool, PO Box 147 Liverpool L 693 BX, G.B.

(4) Laboratoire de Physique des Solides, Université Paris-Sud, 91405 Orsay, France

(Reçu le 7 novembre 1990, accepté le 5 août 199I)

\begin{abstract}
Résumé. - Nous avons étudié trois procédés de texturation des céramiques supraconductrices à haute température critique basés sur: l'alignement de grains paramagnétiques dans un champ magnétique intense, le frittage sous forte charge (frittage-fluage) et la solidification dirigée. Après avoir rappelé le principe et analysé les mécanismes contrôlant chacun de ces procédés, on présente les caractérisations microstructurales, magnétiques et électriques des meilleurs échantillons produits.
\end{abstract}

\begin{abstract}
We have investigated three processes of texturing high $T_{\mathrm{c}}$ superconducting ceramics based on : alignment of paramagnetic grains in a high magnetic field, sintering under a heavy load (creep-sintering) and oriented solidification. After recalling the principle and analyzing the controling mechanisms of each process, microstructural, magnetic and electrical characterization of the best produced samples are presented.
\end{abstract}

\section{Introduction.}

Depuis plus d'un an, nous étudions en collaboration avec les Universités de Liège, de Liverpool et d'Orsay, les possibilités de texturer les céramiques supraconductrices à haute température critique par alignement magnétique, frittage-fluage et solidification dirigée.

\section{Alignement magnétique.}

On sait que placés dans un champ magnétique intense, des grains monocristallins de céramique supraconductrice à haute température critique tournent sur eux-mêmes afin d'aligner leur direction de plus forte aimantation parallèlement au champ [1]. Vu que le tenseur de susceptibilité magnétique des céramiques de type $\mathrm{TRBa}_{2} \mathrm{Cu}_{3} \mathrm{O}_{7}$ est caractérisé par les deux valeurs principales $\chi_{\|_{c}}$ et $\chi_{\perp c}$, les grains, pourvu qu'ils soient libres, aligneront donc 
leur direction $c$ parallèlement au champ lorsque $\chi_{\|_{c}}>\chi_{\perp c}$, c'est le cas de TR $=\mathrm{Y}$, Ho et Dy à la température ambiante, et au contraire perpendiculairement au champ dans la situation opposée, c'est le cas de $\mathrm{TR}=\mathrm{Er}, \mathrm{Eu}, \mathrm{Gd}$.

2.1 ANALYSE DU PHÉNOMÈNE. - Comme nous l'avons montré dans une analyse détaillée du phénomène [2], cette rotation des grains peut être prévue à partir de l'équation fondamentale de la dynamique. Le calcul montre que si au départ tous les grains sont orientés au hasard et que $\Delta \chi=\chi \|_{c}-\chi_{\perp c}$ est positif, alors environ $99,8 \%$ d'entre eux auront leur axe $c$ à moins de $1^{\circ}$ du champ appliqué, et que tous les autres grains seront quasiment orientés au hasard quand $t=10 \tau$. Pour fixer les idées, avec des grains monocristallins dispersés dans une résine époxy de viscosité 10 poises et placés à la température ambiante dans une induction de $1 \mathrm{~T}, \tau$ est d'environ $1 \mathrm{~s}$ pour l'YBaCuO et $0,1 \mathrm{~s}$ pour le $\mathrm{DyBaCuO}$.

2.2 ETUde EXPÉRIMENTAle. - Pour étudier ce phénomène, nous avons dispersé $25 \%$ en poids de poudre de céramique à haute $T_{\mathrm{c}}$ dans $75 \%$ de résine d'enrobage et placé le tout dans un aimant permanent $(B=0,6 \mathrm{~T})$ ou dans une bobine supraconductrice $(B=5$ et $8 \mathrm{~T})$. Après 10 à $15 \mathrm{~min}$, la résine étant polymérisée, nous avons alors effectué des diagrammes de diffraction $\mathrm{X}$ et caractérisé la désorientation des grains par le facteur de qualité

$$
Q=\frac{\text { Intensité de la raie }(110)}{\text { Intensité de la raie }(003)}
$$

$Q=0$ correspondant à une désorientation nulle de tous les grains et $Q=20$ à une désorientation quasiment aléatoire.

En passant de la poudre de $\mathrm{DyBa}_{2} \mathrm{Cu}_{3} \mathrm{O}_{7}$ à travers une série de tamis aux mailles de plus en plus serrées, nous avons observé, toutes choses égales par ailleurs, que la désorientation des grains était d'autant plus faible que le tamis était plus fin (Fig. 1).

Comme d'après l'analyse précédente la rotation des grains est indépendante de leur taille, ce résultat laissait penser que la plupart des grains étaient multicristallins, hypothèse que nous avons vérifiée en observant la poudre en microscopie électronique à balayage. Afin de réduire la désorientation des grains, dans une seconde série d'expériences, nous avons compacté la poudre et refritté le matériau une centaine d'heures à $1000^{\circ} \mathrm{C}$, de manière à obtenir un bloc polycristallin, dont la taille moyenne des grains $(\sim 70 \mu \mathrm{m})$ soit supérieure à celle des mailles du tamis. Puis, nous avons rebroyé l'échantillon en poudre et tamisé cette dernière pour récolter les grains qui traversaient le tamis $20 \mu \mathrm{m}$ mais ne passaient pas à travers celui de $10 \mu \mathrm{m}$. En répétant l'expérience précédente avec cette poudre tamisée, nous avons abaissé le

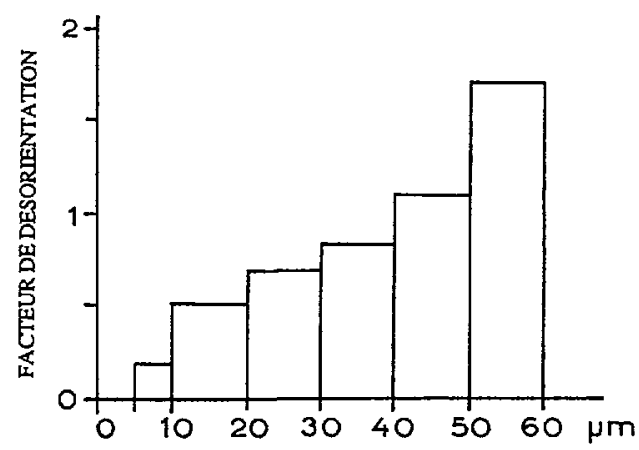

Fig. 1. - Variation du facteur de désorientation en fonction de la taille des mailles du tamis.

[Misorientation coefficient versus the sieve mesh.] 
facteur de désorientation jusqu'à $Q=0,0025$, toujours dans une induction de $0,6 \mathrm{~T}$; mais nous n'avons pu dépasser cette valeur même en utilisant des champs beaucoup plus forts ( 5 et $8 \mathrm{~T}$ ) (Fig. 2). Ceci est vraisemblablement dû au fait que malgré le recuit de grossissement des cristallites, certains grains de poudre sont multicristallins. En effet, à haute température, les cristallites les plus grosses croissent au détriment des plus petites. De ce fait, il subsiste nécessairement des cristallites de très petite taille qui, lors du broyage, restent accolées à des fragments de grosses cristallites et constituent ainsi des grains multicristallins qui passent à travers les mailles des tamis.
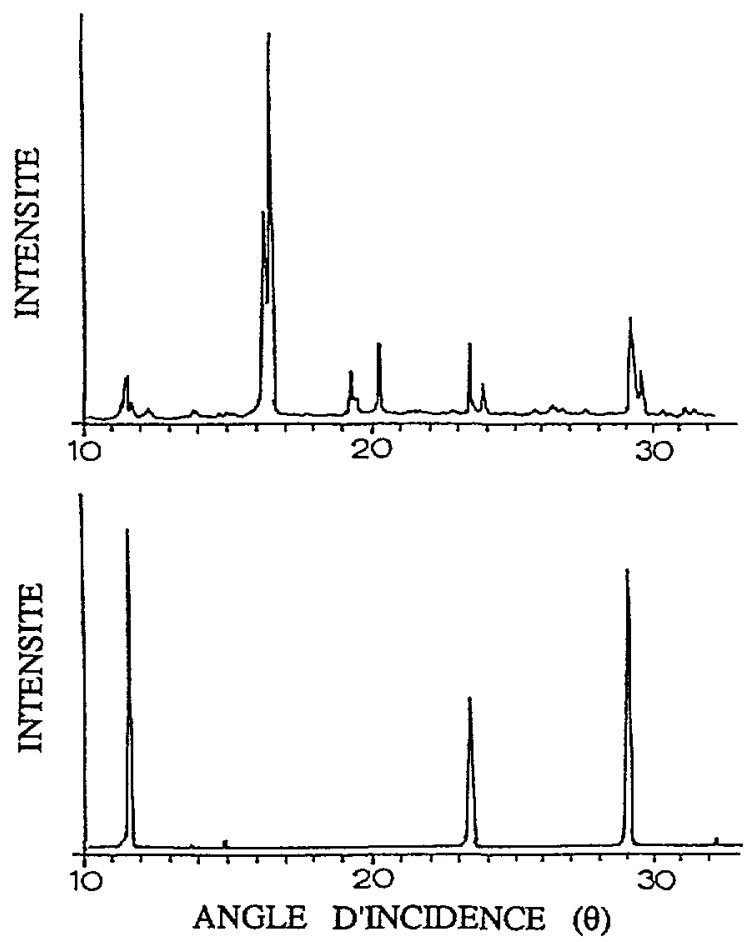

Fig. 2. - Diagrammes de diffraction $\mathrm{X}$ de céramique $\mathrm{DyBa}_{2} \mathrm{Cu}_{3} \mathrm{O}_{7}$. Haut : échantillon de référence non texturé ; Bas : échantillon le plus texturé obtenu par alignement des grains dans un champ de $0,6 \mathrm{~T}$.

[X ray diffraction patterns of $\mathrm{DyBa}_{2} \mathrm{Cu}_{3} \mathrm{O}_{7-\delta}$ ceramics : Up : untextured sample. Down : the best textured sample obtained in a $0.6 \mathrm{~T}$ magnetic field.]

Par contre, comme on pouvait s'y attendre, avec de $\mathrm{l}^{\prime} \mathrm{YBa}_{2} \mathrm{Cu}_{3} \mathrm{O}_{7}$, nous avons trouvé que l'alignement des grains était nettement meilleur à $8 \mathrm{~T}$ qu'à $0,6 \mathrm{~T}(Q=0,04$ et 0,135 respectivement).

Le but de notre étude étant l'obtention de forts courants critiques par texturation du matériau, après avoir ainsi précisé les phénomènes et optimisé la qualité de la poudre, dans une seconde étape nous avons remplacé la résine isolante par différents liquides volatils : acétone, heptane, alcool.. Après évaporation complète de ces derniers, nous avons recueilli une boue sèche que nous avons frittee $12 \mathrm{~h}$ à $920^{\circ} \mathrm{C}$ sous air, puis recuite $48 \mathrm{~h}$ à $400^{\circ} \mathrm{C}$ sous oxygène. Ceci nous a conduit à des échantillons texturés $(0,1 \leqslant Q \leqslant 0,5$ avec $H=$ $0,6 \mathrm{~T})$ qui lévitaient à quelques millimètres au-dessus d'un aimant permanent refroidi à $77 \mathrm{~K}$, mais qui à cause de leur grande porosité étaient trop fragiles pour que l'on en relève les caractéristiques électriques. Nous en avons conclu qu'il était indispensable de compacter les 
grains avant frittage et avons conçu des dispositifs, en cours de réalisation, qui nous permettrons d'effectuer cette opération aussi bien sous champ que hors champ.

\section{Frittage-fluage.}

3.1 PRINCIPE DE LA MÉTHODE. - Nous appelons frittage-fluage l'opération qui consiste à fritter un échantillon sous une charge telle qu'il s'écrase abondamment en même temps qu'il se fritte. En l'absence de charge appliquée, on sait que la densification forte et rapide des céramiques à haute $T_{\mathrm{c}}$, que l'on observe au-dessus de $900^{\circ} \mathrm{C}$, est due à la présence d'une phase liquide qui accélère les processus de frittage et qui confère aux grains leur forme de plaquettes parallèles au plan de base $(a, b)$ de la maille orthorhombique de leur structure cristalline. Par conséquent, si l'on écrase lentement les échantillons durant cette phase de frittage, il est clair que les grains, lubrifiés et entraînés par la phase liquide, rouleront et glisseront les uns sur les autres de manière à construire une structure ressemblant à un mur de briques, dans laquelle les plans conducteurs $a, b$ seront alignés. Mais il est aussi évident que la phase liquide accélérera la formation des ponts entre les grains qui s'opposeront à la rotation et au glissement de ces derniers. Par conséquent, la texturation des céramiques supraconductrices par frittage-fluage apparaît comme le résultat de deux tendances opposées et nécessite une optimisation pointue pour obtenir un haut niveau de texture.

3.2 OPTIMISATION DU PROCÉDÉ. - Afin d'obtenir les textures souhaitées, nous nous sommes efforcés d'optimiser le cycle de température et de charge représenté sur la figure 3, en conservant constants les paramètres suivants: $\stackrel{\circ}{T}_{1}=600^{\circ} \mathrm{C} / \mathrm{h}, T_{1}=650^{\circ} \mathrm{C}, \Delta t_{1}=0,5 \mathrm{~h}$, $\Delta t_{2}=1 \mathrm{~h}, \stackrel{\circ}{T}_{3}=60^{\circ} \mathrm{C} / \mathrm{h}, L_{1}=10 \mathrm{~kg}$ et sauf avis contraire, la vitesse d'écrasement $S$ durant la montée en charge. Tous les autres paramètres ont été variés systématiquement un à la fois.

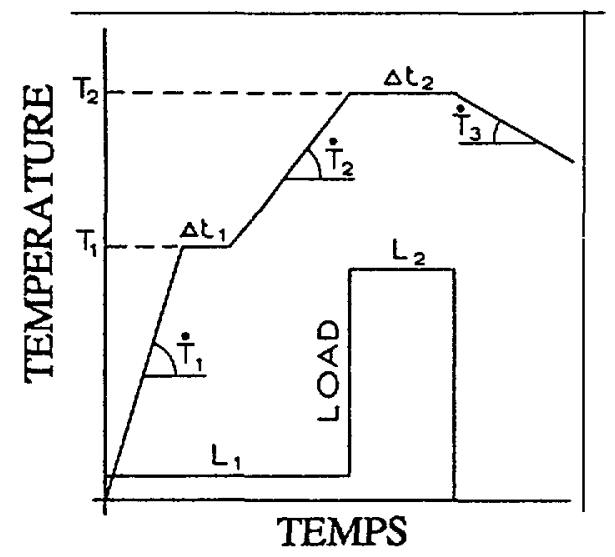

Fig. 3. - Cycle de température et de charge.

[Temperature and load schedule.]

Sur le plan pratique, nous avons découpé des tranches de $10 \mathrm{~mm}$ d'épaisseur dans des barreaux d'YBaCuO de $16 \mathrm{~mm}$ de diamètre obtenus par pressage à froid. Ces tranches prises en sandwich entre deux feuilles d'argent doublées de mica furent insérées dans les mâchoires d'une presse à chaud pour être frittées sous charge. Comme le montre la figure 4, pour une température de frittage donnée $\left(920^{\circ} \mathrm{C}\right)$, la désorientation des grains est d'autant plus faible que la charge appliquée est plus lourde. Mais, plus que la charge elle-même, ce qui importe 


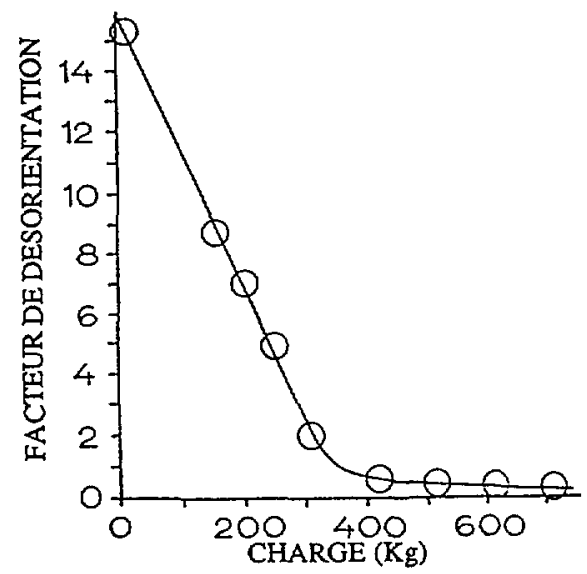

Fig. 4. - Influence de la charge sur la désorientation.

[Misorientation versus load.]

est le taux de déformation qu'elle impose à l'échantillon. En effet, même en faisant varier d'autres paramètres $\left(\stackrel{\circ}{T}_{2}, T_{2}, \Delta t_{2}\right.$, . ) nous avons constaté (cf. Fig. 5) que dans la représentation facteur de qualité - taux de déformation ( $\tau=$ épaisseur initiale/épaisseur finale), les points représentatifs de tous nos échantillons se situaient sur une même courbe et qu'il fallait réduire l'épaisseur des échantillons d'un facteur 10 environ pour obtenir une texture très prononcée à la fin du cycle schématisé sur la figure 1 .

Les courbes de fluage que nous avons enregistrées montrent que la plus large part de la déformation s'effectue durant la courte période pendant laquelle on augmente la charge jusqu'à sa valeur nominale, mais que l'échantillon continue de se déformer à une vitesse de plus en plus lente tout au long du palier de frittage. Pour approfondir la compréhension des mécanismes de texturation, il importe donc de savoir quand se construit la texture : durant le court stade de déformation rapide ou au contraire au cours du long stade de fluage proprement dit. Afin d'éclairer ce point, nous avons effectué deux séries d'expériences. Dans la première, lors de la mise en charge, les échantillons furent écrasés à différentes vitesses

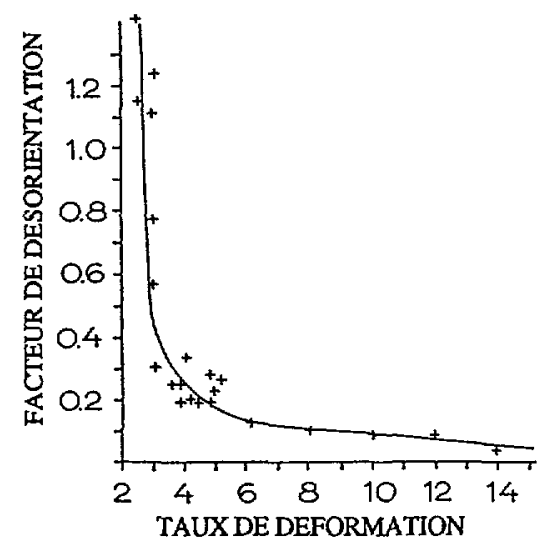

Fig. 5. - Influence du taux de déformation sur la désorientation des grains.

[Misorientation versus deformation rate.] 
jusqu'à ce que le taux de déformation atteigne 2,5 , puis la charge fût enlevée immédiatement et les échantillons sortis du four aussi vite que possible. Les diagrammes de diffraction $\mathrm{X}$ effectués sur ces échantillons montrent clairement qu'à la fin de ce stade de déformation rapide les échantillons sont déjà notablement texturés : $Q \sim 1$ si la vitesse de déformation est comprise entre 0,2 et $1 \mathrm{~mm} / \mathrm{min}$. Aux vitesses inférieures les ponts formés entre les grains durant la montée en température se consolident plus vite par frittage, qu'ils ne se déforment par fluage, ce qui freine la rotation des grains et réduit leur alignement. Aux vitesses supérieures, nous pensons que le transport de matière par la phase liquide n'est plus assez rapide pour donner aux grains leur forme de plaquette, ceux-ci se rangent donc de façon plus aléatoire et de ce fait, la texture est moins prononcée. Par ailleurs, nous avons remarqué que dans ce stade de déformation rapide la vitesse de déformation est proportionnelle à la contrainte appliquée à une puissance égale à 1 aux erreurs expérimentales près. Ceci indique que la vitesse n'est pas contrôlée par le glissement et la montée des dislocations car elle serait alors proportionnelle à la contrainte appliquée à une puissance comprise entre 3,5 et 7 , mais par un mécanisme d'écoulement visqueux.

Dans la seconde série d'expériences, nous avons conservé la vitesse d'écrasement constante $(5 \mathrm{~mm} / \mathrm{min}$ ) jusqu'à ce que la charge atteigne $300 \mathrm{~kg}$ et avons alors asservie la vitesse pour maintenir la charge constante. En utilisant un nouvel échantillon à chaque fois, nous avons observé la progression de la texture en fonction de la durée du frittage sous contrainte (Fig. 6). Cette courbe traduit assez bien le ralentissement rapide de la vitesse de déformation en fluage et corrobore la corrélation entre le facteur de désorientation et le taux de déformation (cf. Fig. 5).

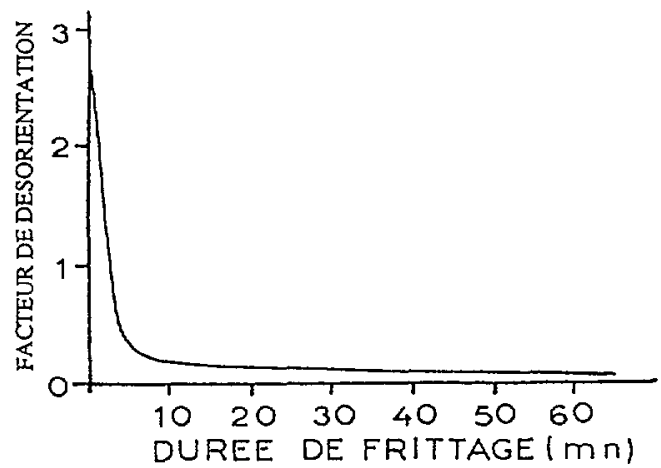

Fig. 6. - Evolution de la désorientation en fonction de la durée de frittage.

[Misorientation versus sintering time.]

L'effet de la température de frittage sur l'alignement des grains (Fig. 7) confirme pleinement l'idée d'un frittage en présence d'une phase liquide. En effet, pour une charge donnée $(310 \mathrm{~kg})$, nous avons observé qu'en dessous de $900{ }^{\circ} \mathrm{C}$ l'échantillon ne se déformait quasiment pas et par suite ne se texturait pas $(Q>8)$, tandis que dès $910^{\circ} \mathrm{C}$ il commençait à se déformer et à se texturer $(Q \sim 3)$ et qu'à $920^{\circ} \mathrm{C}$ la déformation et la texture étaient très prononcées $(Q \leqslant 0,5)$. Il est clair qu'une dépendance aussi brutale de la vitesse de déformation avec la température est en parfait accord avec l'apparition d'une phase liquide vers $900^{\circ} \mathrm{C}$ et ne correspond à aucun mécanisme thermiquement activé connu. A plus haute température, la texture est moins bonne car la déformation est moindre vu que le pourcentage de phases secondaires augmente à partir de $920^{\circ} \mathrm{C}$, que 1'on applique ou non une contrainte sur l'échantillon [3]. 


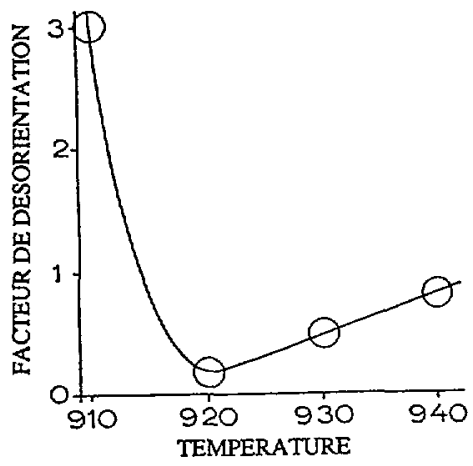

Fig. 7. - Influence de la température de frittage sur la désorientation des grains.

[Misorientation versus sintering temperature.]

D'une manière plus générale, comme nous l'avons montré dans des publications antérieures $[4,5]$, l'influence sur la qualité de la texture, de tous les autres paramètres : vitesse de montée en température ; instant auquel on applique la charge; nature du matériau de contact.. est en bon accord avec l'hypothèse d'un frittage en présence d'une phase liquide.

3.3 Caractérisation microstructurale. - Nous avons systématiquement observé en microscopie optique des sections polies de nos échantillons, tant parallèles que perpendiculai-

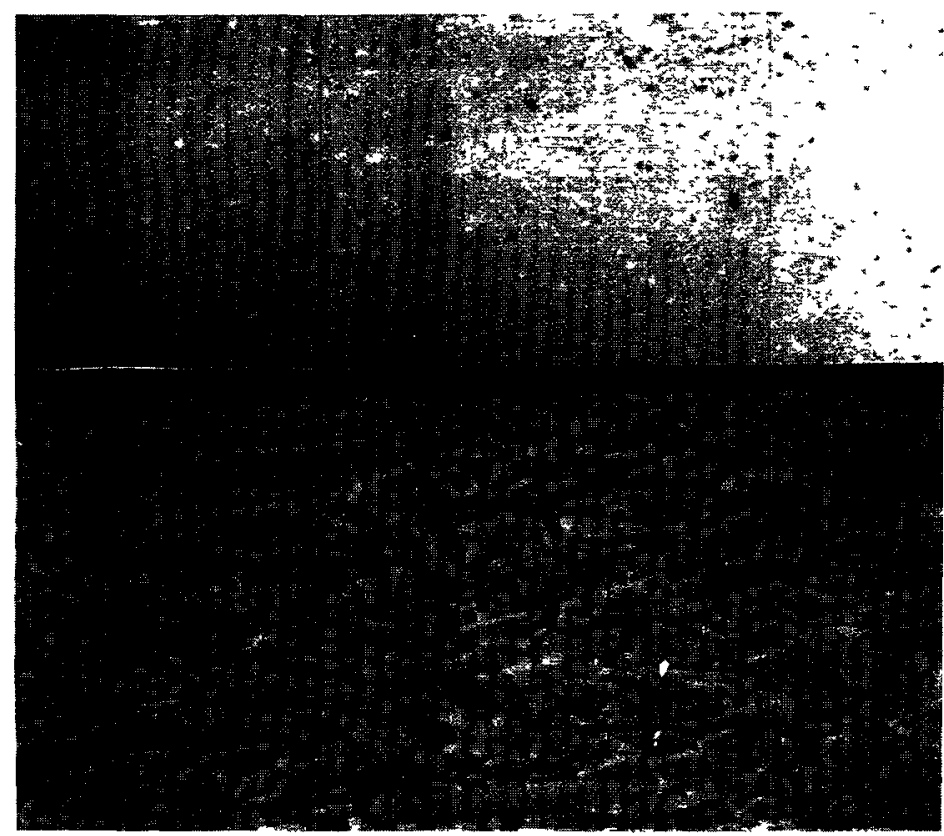

Fig. 8. - Aspect d'une coupe parallèle à la charge appliquée d'un échantillon texturé à $Q=0,05$ préparé avec de la poudre RP : a) microscopie optique lumière blanche, b) microscope à balayage après gravage des joints.

[Microstructure parallel to the load for a sample with $Q=0.05$. Rhone-Poulenc precursors. a) Optical microscope observation. b) SEM observation after etching.] 
res à la charge appliquée. En lumière ordinaire, sur tous les échantillons préparés à partir d'YBaCuO Rhône-Poulenc, on distingue seulement quelques grains brillants et d'autres plus petits et noirs que nous avons identifiés à la microsonde électronique comme étant respectivement du $\mathrm{CuO}$ et du $\mathrm{BaCuO}_{2}$. Ces échantillons semblent parfaitement compacts car aucun pore n'est visible à cette échelle (Fig. 8a). Au contraire avec la poudre P9, préparée au laboratoire, des pores sont visibles mais la porosité n'excède pas quelques \% (Fig. 8b). Ceci se comprend bien car la poudre RP est fine $(\sim 1 \mu \mathrm{m})$ incomplètement, réagie et carbonatée, tandis que la poudre P9 est grosse $(\sim 5 \mu \mathrm{m})$ et, nettement plus monophasée. Par suite, il y a plus de phase liquide avec la poudre RP qu'avec la poudre P9 et très logiquement la densification est plus forte avec RP qu'avec P9. En lumière polarisée les grains apparaissent sous forme de plaquettes, maclées ou non suivant le degré d'oxygénation, mais pour mieux révéler leur contour, nous avons gravé les joints de grains par attaque métallographique, ce qui permet en plus de les observer à fort grossissement au microscope à balayage. En optimisant les divers paramètres, avec la poudre RP nous avons atteint un facteur de qualité

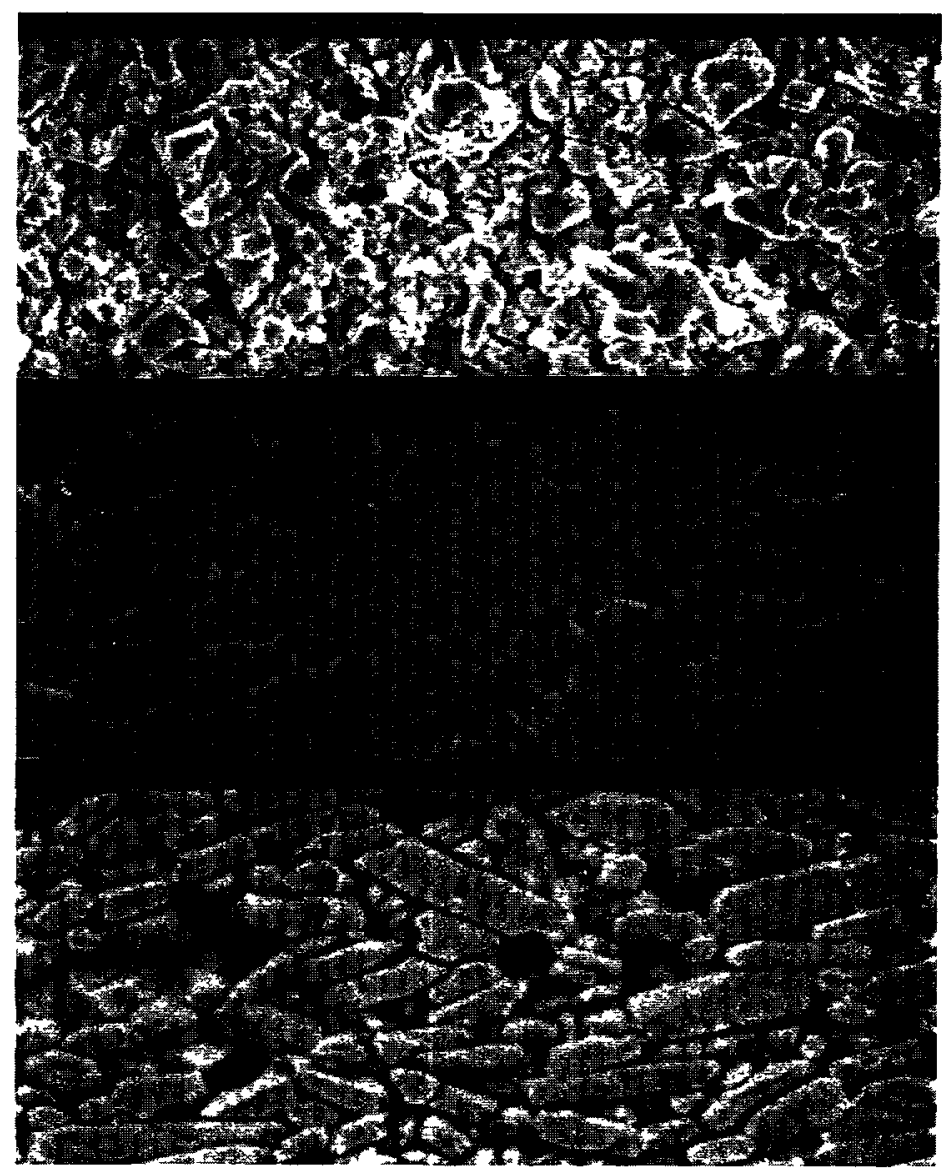

Fig. 9. - Observation en microscopie électronique à balayage d'une coupe parallèle à la charge appliquée d'un échantillon texturé à $Q=0,5$ préparé avec la poudre $\mathrm{P} 9:$ a) poudre compactée avant frittage, b) aspect après frittage sans gravage des joints, c) aspect après frittage et gravage des joints.

[SEM observations of microstructures parallel to the load for samples with $Q=0.5$. P9 powder. a) Green ceramic. b) After sintering, without etching. c) After sintering, with etching.] 
de 0,05 ce qui correspond à une désorientation de quelques degrés seulement entre les grains (cf. Fig. 8b).

Dans les mêmes conditions, avec la poudre P9, nous n'avons abaissé le facteur de désorientation que jusqu'à 0,5 . Comme le montrent les figures $9 \mathrm{a}, \mathrm{b}$ et c pour $\mathrm{P} 9$, mais cela reste vrai pour RP, le frittage-fluage s'accompagne d'une forte croissance des grains surtout de ceux qui sont les mieux orientés.

Au vu de ces observations, nous pensons que la texture se construit comme suit. Quand la phase liquide apparaît, elle mouille la surface des grains, au moins en partie, et s'accumule à leurs points de contact. Dès lors, les plus petits grains se dissolvent progressivement et la matière dissoute se redépose sur les plus gros. Ce phénomène, appelé coalescence, étant contrôlé par la diffusion dans une phase liquide, le flux de matière ainsi transportée est considérable et comme la vitesse de dépôt est très anisotrope, on conçoit que les grains acquièrent rapidement leur forme de plaquette parallèle aux plans $a, b$. A priori ces plaquettes sont rangées au hasard, mais en appliquant une contrainte uniaxiale, on les fait glisser et tourner sur elles-mêmes de manière à ce qu'elles forment une structure ressemblant à un mur de briques. Nous nous efforçons actuellement de modéliser ce phénomène très complexe.

3.4 Caractérisation Électrique et magnétique. - En diffraction $X$, nous avons observé que bruts de frittage sous charge, tous les échantillons préparés avec la poudre RP ont une stœchiométrie en oxygène inférieure à 6,5 et de ce fait ne sont pas supraconducteurs, même à 4,2 K (d'après les mesures de susceptibilité alternative). Il est donc nécessaire de les réoxygéner. Mais probablement à cause de leur très forte densité cette opération est particulièrement difficile et nous n'y sommes parvenus qu'après un traitement de grossissement des grains qui a développé de la porosité secondaire. Ainsi traités, ces échantillons présentent une transition résistive très étroite à $92 \mathrm{~K}[6]$ et une anisotropie de susceptibilité magnétique comparable à celle observée sur les monocristaux (Fig. 10). Mais malgré la texture la densité critique de courant n'est que de $160 \mathrm{~A} / \mathrm{cm}^{2}$ à l'azote liquide car de nombreux joints sont soit fissurés soit bordés de phase amorphe provenant de la solidification de la phase liquide comme l'ont montré les observations en microscopie électronique en transmission.

$\mathrm{Au}$ contraire, bruts de frittage sous charge, les échantillons préparés avec la poudre P9 sont déjà légèrement supraconducteurs à $77 \mathrm{~K}$ et se réoxygènent raisonnablement bien. Les mesures de résistivité effectuées en courant continu sur des barreaux de $1,5 \times 1,5 \times$

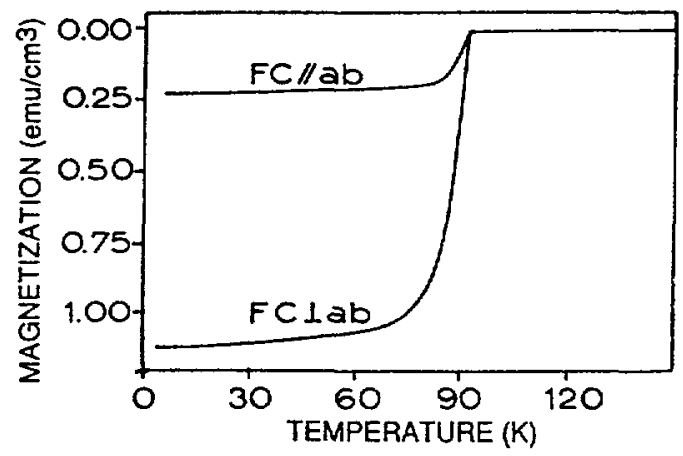

Fig. 10. - Variation de la magnétisation en fonction de la température mesurée en refroidissant les échantillons sous champ.

[Magnetization versus temperature during cooling in a magnetic field.] 


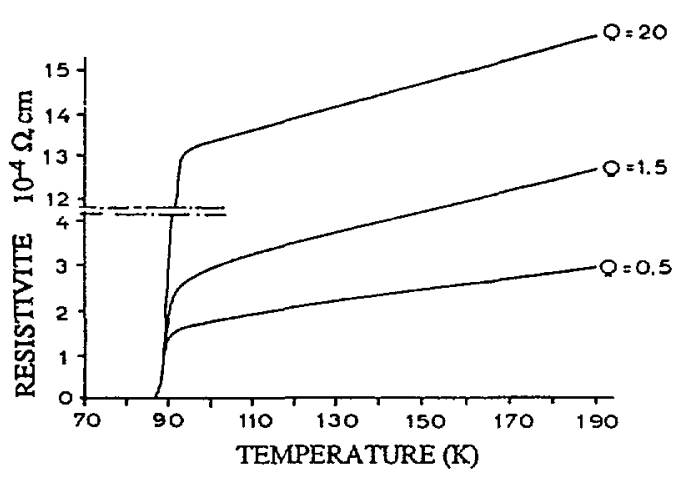

Fig. 11. - Variation de la résistivité en fonction de la température d'échantillons présentant des facteurs de désorientation différents.

[Resistivity versus temperature for samples with different $Q$ values.]

$20 \mathrm{~mm}^{3}$ par la méthode dite 4 points, montrent que dans le domaine normal la résistivité de nos échantillons est d'autant plus basse qu'ils sont plus texturés et que la transition résistive est d'abord très brutale vers $85 \mathrm{~K}$ puis beaucoup plus douce avant de s'annuler vers $80 \mathrm{~K}$ (Fig. 11). De ce fait le courant critique reste modeste : $J_{c}=600 \mathrm{~A} / \mathrm{cm}^{2}$ à $63 \mathrm{~K}$ mesuré sur l'échantillon présentant un facteur de désorientation de 1,5. En améliorant la texture, l'homogénéité du matériau et surtout la réoxygénation nous espérons largement dépasser ces valeurs.

Par ailleurs, nous avons obtenu des premiers résultats très encourageants avec les composés au bismuth qui ne nécessitent pas de réoxygénation.

\section{Solidification dirigée.}

Tous les supraconducteurs à haute $T_{\mathrm{c}}$ ont une fusion non congruante. Par conséquent, en portant ces matériaux au-dessus de leur point de fusion péritectique, on obtient une importante quantité de liquide en équilibre avec une quantité relativement faible de phase solide non supraconductrice (la phase verte 211 dans le cas des TRBaCuO). Si l'on refroidit très lentement le liquide dans des conditions très isothermes la vitesse de germination de la phase solide supraconductrice devient si lente que tout le liquide se solidifie sur les quelques germes formés et l'on obtient ainsi quelques gros cristaux. C'est la technique classique de préparation des petits monocristaux de supraconducteurs à haute $T_{\mathrm{c}}$ par la méthode dite du flux (on s'écarte volontairement de la composition de la phase supraconductrice pour conserver de la phase liquide en équilibre avec la phase solide). Au contraire, si on refroidit l'échantillon dans un faible gradient de température que l'on déplace lentement, on conserve une faible vitesse de germination et on oriente la croissance des grains dans le gradient de température. C'est la technique dite de melt texturing qui produit des échantillons fortement texturés présentant une densité critique de courant élevée [7]. Si le gradient est encore plus faible et qu'on le déplace encore plus lentement toute la phase liquide se solidifie sur le premier germe formé et on obtient une matrice monocristalline dans laquelle sont dispersés des grains de la phase non supraconductrice stable à haute température et le résidu de la phase liquide solidifiée. C'est la technique mise au point par nos partenaires de l'Université de Liverpool à partir des barreaux frittés d'YBaCuO que nous leur fournissons dans le cadre du contrat Brite/EURAM BE $0067 \mathrm{C}$ (EDB) dont nous sommes le leader. 
4.1 Caractérisation microstructurale. - Les barreaux qui ont 1,5 à $2 \mathrm{~mm}$ de diamètre sont monocristallins sur une longueur de $40 \mathrm{~mm}$. Comme l'orientation du premier germe est aléatoire les plans conducteurs $a, b$ sont généralement écartés de l'axe du barreau, typiquement 20 à $60^{\circ}$ dans les quelques échantillons préparés jusqu'à présent. Sur une coupe métallographique, on constate la même orientation sur toute la longueur de l'échantillon $(40 \mathrm{~mm})$. On observe de plus que les grains de phase verte 211 sont petits, très arrondis, qu'ils ne percolent pas et qu'ils n'occupent guère plus de 5 à $10 \%$ du volume. Quant au résidu de phase liquide qui s'est solidifié en dernier, il apparaît sous forme de strates discontinues distantes de 20 à $30 \mu \mathrm{m}$ et parallèles aux plans conducteurs.

4.2 Caractérisation Électrique et magnétique. - En mesurant la chute de potentiel en fonction du courant continu traversant de façon permanente l'échantillon, jusqu'à près de $100 \mathrm{~A}$, nous avons déterminé que la densité critique de courant transporté correspondant à une chute de $1 \mu \mathrm{V} / \mathrm{cm}$ était de $7000 \mathrm{~A} / \mathrm{cm}^{2}$ à $77 \mathrm{~K}$ suivant l'axe du barreau. Par mesures magnétiques [8] rapportées à la longueur du barreau, nous avons confirmé cette valeur. De plus, en découpant des tranches d'échantillon parallèles aux plans conducteurs, nous avons déterminé le courant critique tant suivant l'axe $c$ que dans le plan $(a, b)$ par mesure magnétique en fonction de la température et du champ appliqué (Fig. 12).

En champ nul, la densité critique de courant dans le plan $(a, b)$ atteint $10^{5} \mathrm{~A} / \mathrm{cm}^{2}$ à $77 \mathrm{~K}$ et dépasse probablement $2 \times 10^{6} \mathrm{~A} / \mathrm{cm}^{2}$ à $4,2 \mathrm{~K}$, valeurs qui sont les meilleures rapportées actuellement sur des monocristaux massifs. Suivant l'axe $c$, ces valeurs tombent respectivement à $4 \times 10^{4}$ et $3 \times 10^{5} \mathrm{~A} / \mathrm{cm}^{2}$ ce qui reste très élevé.

Ce dernier faisceau de courbes montre la très bonne tenue au champ, au moins jusqu'à $3 \mathrm{~T}$, de la densité critique de courant de nos échantillons, aussi bien suivant l'axe $c$ que le long des plans $a, b$. On voit donc que ces échantillons de grande taille ont des caractéristiques égales et même supérieures à celles mesurées sur les meilleurs monocristaux de petite taille préparés jusqu'alors.

Finalement les mesures de susceptibilité alternative effectuées à l'Université de Liège montrent que le courant critique des échantillons de ce type n'est pas limité par des problèmes de jonction faible car les courbes donnant la partie réelle et la partie imaginaire de la susceptibilité alternative en fonction de la température ne se déplacent pas vers les hautes températures quand on réduit le champ alternatif utilisé pour les mesures.

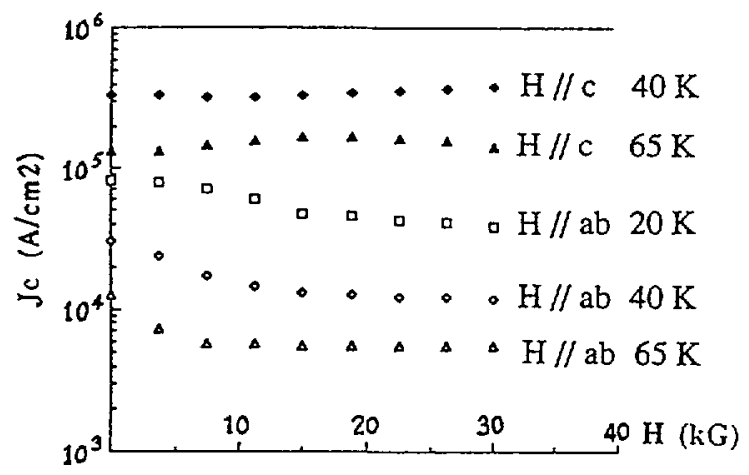

Fig. 12. - Variation de la densité critique de courant en fonction du champ appliqué parallèlement à $c$ ou parallèlement au plan $(a, b)$ à différentes températures.

[ $J_{\mathrm{c}}$ versus magnetic field.] 


\section{Conclusion.}

Grâce à une bonne coopération entre physiciens du solide et scientifiques des matériaux, nous avons rapidement fait progressé notre étude sur la texturation des supraconducteurs à haute température critique. En poursuivant cet effort, nous espérons mieux comprendre tant les mécanismes qui contrôlent la formation des textures dans ces matériaux que les raisons physiques qui en limitent la densité critique de courant, ce qui devrait permettre d'optimiser ces matériaux pour les applications pratiques.

\section{Remerciements.}

Nous remercions la Communauté Economique Européenne pour le large soutien financier qu'elle nous accorde, l'Ecole des Mines d'Alès pour l'attribution d'une bourse ARMINE à Mr Deschanels, ancien élève de l'Ecole et le Comptoir Lyon Alemand Louyot pour le cofinancement de la bourse CIRCEA de Mr Chaffron. Nous remercions aussi Mrs Lesmond et Alloul de nous avoir prêté leurs bobines supraconductrices.

\section{Bibliographie}

[1] Farell D. E. and Chandrasekhar B. S., Phys. Rev. B 36 (1987) 4025.

[2] REgnier P., Deschanels X. and Faisant J. P., à paraître.

[3] Keating S. J., Wei I. and Chien T. Y., Ceramic Supraconductor II, Am. Ceram. Soc. (1988) $43-$ 50.

[4] Régnier P., Le Hazif R. and Chaffron L., Proceeding of the Int. Conf. on Modern Aspects of Superconductivity IITT International publication (Paris, 23-24 novembre 1989) pp. 133-138.

[5] RÉgnier P., Proceeding of the 7th CIMTEC World Ceramic Congress Satellite Conf. 4 HTC Superconductors (Trieste, 2-5 juillet 1990).

[6] Bonjour E, et al., Physica C 166 (1990) 451-455.

[7] Jin S., Sherwood R. C., Van Dover R. B., Tiefel T. H. and Johnson Jr D. W., Appl. Phys. Lett. 51 (1987) 203-204.

[8] Aguillon C., McCartney D. G., Régnier P. and Senoussi S., Submitted for publication to $J$. Appl. Phys. 